\title{
Hands-On Release Planning with Poker Chips
}

\author{
Jason Yip, ThoughtWorks, Inc. \\ jcyip@thoughtworks.com
}

You've gathered enough user stories or features that you're now interested in figuring out what to include in the next release. You have a reasonable view on how large everything is and how many people will be available, though of course at this stage of the game there is a reasonable likelihood of variation.

This is the point in which you engage in the practice of Release Planning.

Although this paper is not about Release Planning, it's worth providing a quick summary for those unfamiliar with the practice. The essence of Release Planning [Wells, 1999] is quite simple:

- Titles of user stories [Cohn, 2004], which are a way of describing features, are written or printed onto index cards.

- Each of these story cards will be associated with an estimate, typically based on relative sizing.

- The story cards are laid out on a large table.

- Stakeholders negotiate and select which stories should be included in the first release.

This paper is about a subtle adjustment to how that last item is done and why that adjustment is worth doing.

\section{When engaging in Release Planning, use emotionally engaging physical tokens to represent points that can be allocated to user stories.}

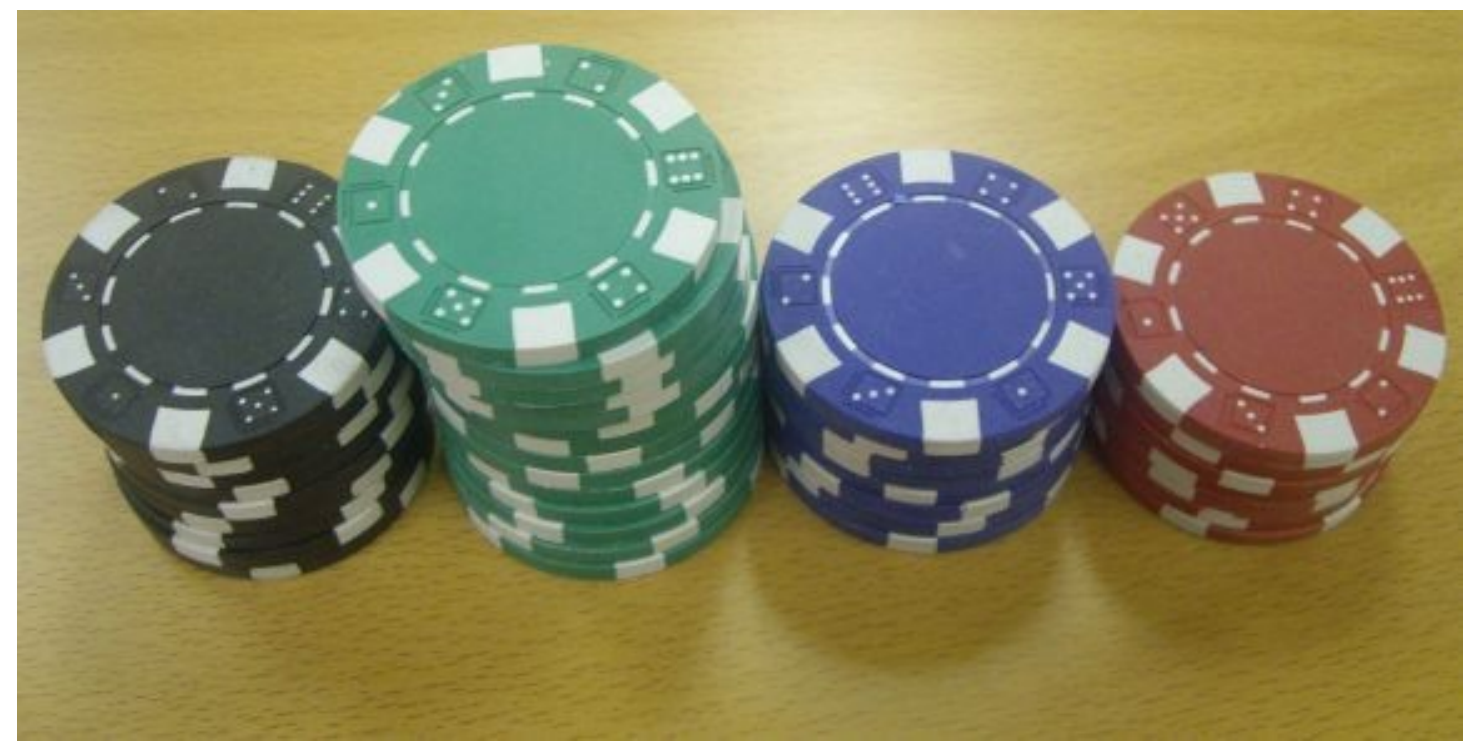

\section{Physical metaphors and models are more intuitive than abstract numbers}

You can't put $11 \mathrm{lbs}$ of... uh, dirt... in a $10 \mathrm{lb}$ bag. This physical metaphor was used in the original first edition of the Extreme Programming book [Beck, 1999] to communicate the concept of planning according to capacity. The metaphor works because it's something you can visualise; something you can imagine even if you haven't directly experienced it.

But what if instead of a conceptual metaphor, you use concrete, physical objects? Instead of 


\section{Planning authority and responsibility should be kept in the hands of stakeholders}

"Product managers", "Project owners", "Project sponsors", etc. are presumably involved in the project because they have the authority to decide what is the appropriate set of features for the system. In many cases, this may be a team of people including a more technical Chief Architect/Engineer that decides what is appropriate.

This is not to say that project team members should not provide information and advice but the key point is that it is not the authority or responsibility of the engineering team to override decision-making responsibility for scoping.

\section{Boredom leads to low involvement; Low involvement leads to low commitment}

The reality is that sometimes work is uninteresting. But many times it's how the activity is designed. Instead of trying to convince people to be engaged with an uninteresting activity, we can instead deliberately design the activity to be engaging.

\section{To summarise...}

- Physical models emotionally convey the limits of resources and time much better than words or numbers

- The authority and responsibility of scope inclusion should be with the business stakeholders, not the engineering team

- A boring release planning process will tend to have less engagement and commitment from stakeholders

\section{Therefore...}

\section{Use poker chips in Release Planning to emotionally engage stakeholders}

Provide chips to the stakeholders reflecting the capacity of the engineering team for the release. Ask the stakeholders to purchase stories for the release by placing the appropriate number of chips on story cards, 1 chip for each story point. For example, 3 chips would need to be placed to purchase a 3 point user story.

Different colour chips can be used to allow for multiple estimation contexts. For example, white chips represent base capacity while blue chips represent potential excess capacity if the team is faster than expected.

\section{Before chip placement}




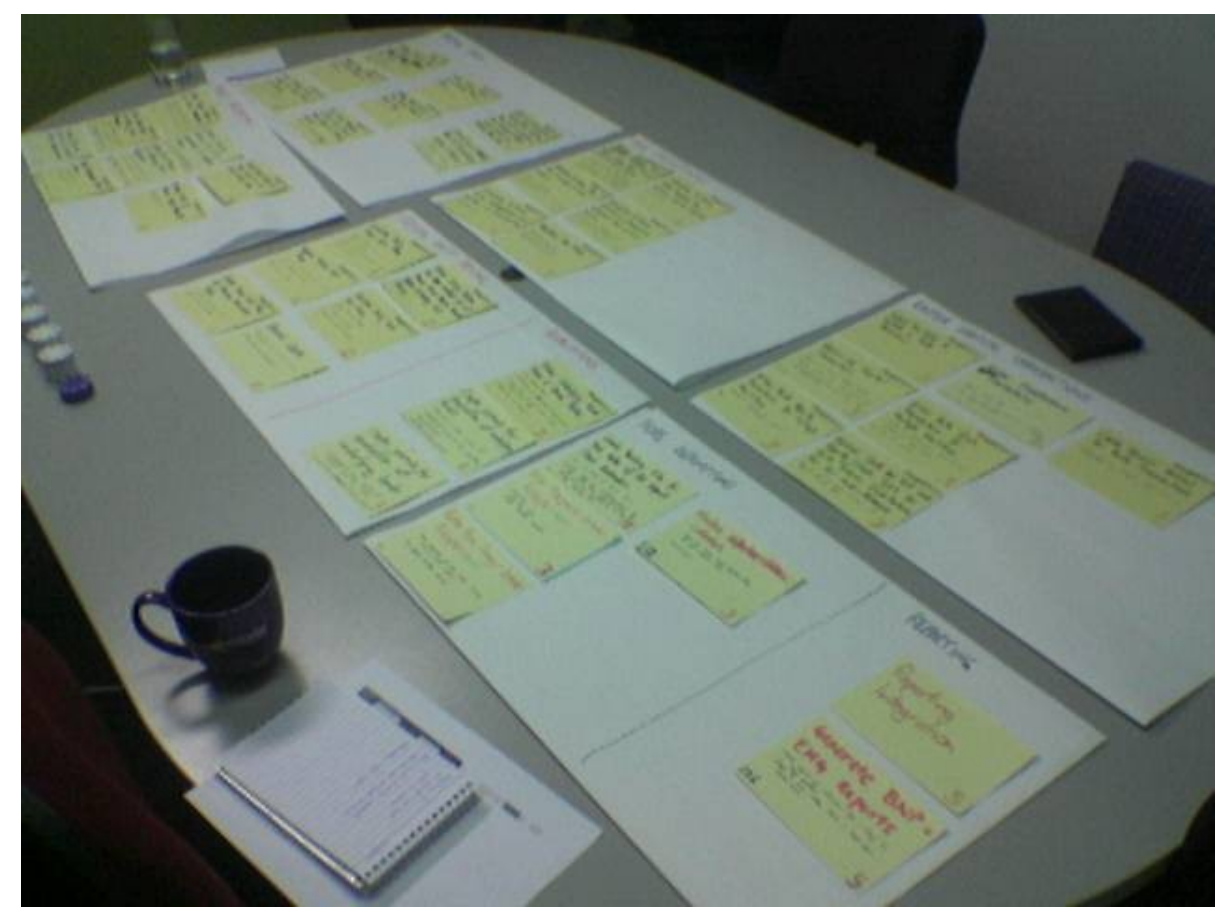

\section{After chip placement}

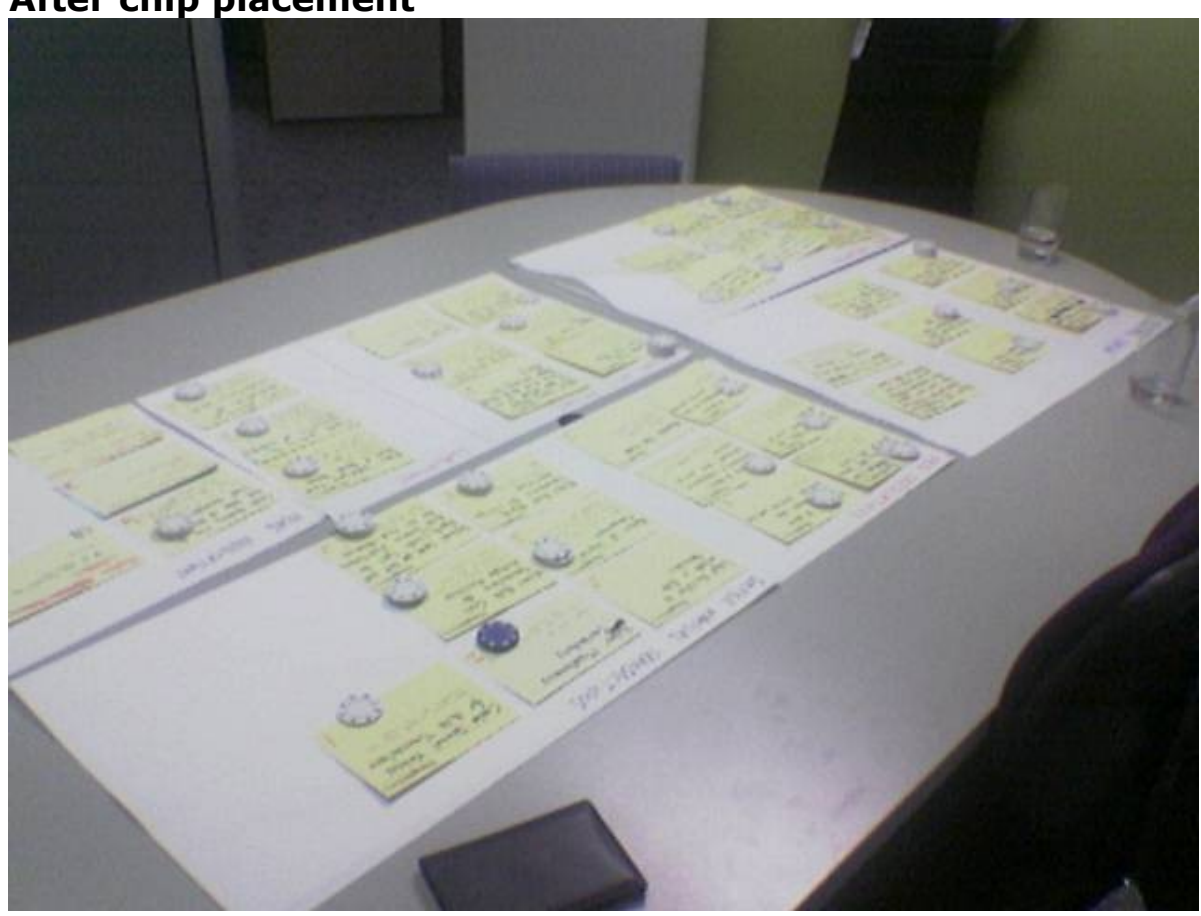

Physical tokens, such as poker chips, provide a more visceral experience to planning. Planning becomes something that engages the senses. The sense of texture from holding a clay poker chip, the distinct click of placing a chip on a story card, all these things are very effective in changing the presence of an otherwise mundane activity. When you see stakeholders start playing with the chips while deciding which story to select next, you realise how much the experience has been changed.

The act of spending something to purchase something is a concept that business people can readily accept. Ironically real coins feel cheap because their associated value is too low. Real coins also tend to be rather dirty and are not as easy to differentiate as coloured chips. 


\section{It's not really about poker chips}

The point is not about using poker chips. The point is that using physical tokens that appeal to your stakeholders will increase the level of engagement in the release planning process. The specific type of token that will be considered appealing varies based on cultural and organisational context.

Tokens build upon the pre-existing physically engaging nature of release planning with index cards. Physical engagement is used to increase mental engagement.

So it's not really about poker chips; poker chips are simply the best expression that I've seen so far.

\section{It's not just the tokens; it's also the facilitation approach}

Standard facilitation approaches for release planning sessions still apply. We still want the stakeholders to take lead on resolving conflicts amongst themselves. We still want to be explicit up front about preallocated points due to technical or other reasons to avoid disappointment. We still want to provide advice and reminders of project goals if the process gets stuck and is otherwise unable to get unstuck on its own.

\section{I'd like to end with a story}

The poker chips worked well - as we'd used coins previously with M. they were already comfortable with the idea of "spending" story points by placing tokens on the cards (in fact $A$. pretty much took the lead in explaining to the business participants who hadn't been there last time around how the process worked) and enjoyed the very hands-on feel it gives.

The chips have a satisfying heft and a gratifying sound when you pop them on the table both good, but the killer for me was having the colours; we had three team sizes we were considering for the release and we used white, blue and red chips to indicate the base story points and two levels of increment (e.g. you get 70 base SPs [white], 13 extra SPS for a slightly larger team [blue], and 13 more SPs for the largest team size [red]).

Having the colours gave us a level of visual prioritisation, i.e. stories paid for by red chips would fail to make the cut first, followed by blue and then white. On a couple of occasions cards were demoted and others promoted by having their chip colour swapped. We probably didn't exploit this as much as we could have done but it was a dimension that I thought was (and has potential to be) very helpful - coins or other undifferentiated tokens can't support this.

On the downside the chips in their case are remarkably heavy and the case itself looks like it contains a sniper rifle (of course this could be good if i) you want a work-out ii) you like intimidating other people in the street) :) 


\section{Acknowledgements}

I'd like to thank Linda Rising for shepherding this paper for PLoP 2007.

\section{References}

[Beck, 1999] Beck, K. Extreme Programming Explained, Addison-Wesley

[Cohn, 2004] Cohn, M. User Stories Applied: For Agile Software Development, Addison-Wesley

[Wells, 1999] Wells, D. "Release Planning", Extreme Programming: A gentle introduction, 1999, URL: http://www.extremeprogramming.org/rules/planninggame.html 